\title{
INTRAVENOUS FLUID MANAGEMENT. DO WE REALLY KNOW WHAT WE PRESCRIBE?
}

\section{Baños Lapuente V., Merten A., Hoffmann R., Casas Vilá J.I., Bastitta M., Moral García M.V. DEPARTMENT OF ANAESTHESIOLOGY. SANT PAU AND SANTA CREU HOSPITAL, BARCELONA}

\section{Background}

- At our tertiary university hospital, more than 250.000 litres of intravenous (iv) cristalloid and colloid solutions are prescribed every year to all type of patients.

- Administration of iv solutions is part of everyday praxis and it is also part of many protocols (sepsis, polytrauma or massive bleeding).

- Most of the inpatients receive the same type of fluid regime despite differences in age, weight, individual cardiac reserve or cause of acute admission.

\section{Material and methods}

- We designed a questionnaire in order to find out if the professionals prescribing and administering iv solutions were aware of the content of the solutions available at our hospital: normal saline (NS), isotonic glucose saline, $5 \%$ dextrose, $10 \%$ dextrose modified fluid gelatine (GFN®) and plasma-lyte balanced crystalloid (PLY 7.4®).

- They were asked about sodium or glucose content, $\mathrm{pH}$, osmolarity, and about the presence of other electrolytes such as potassium, magnesium or calcium.

320 people answered the questionnaire $(100 \%)$
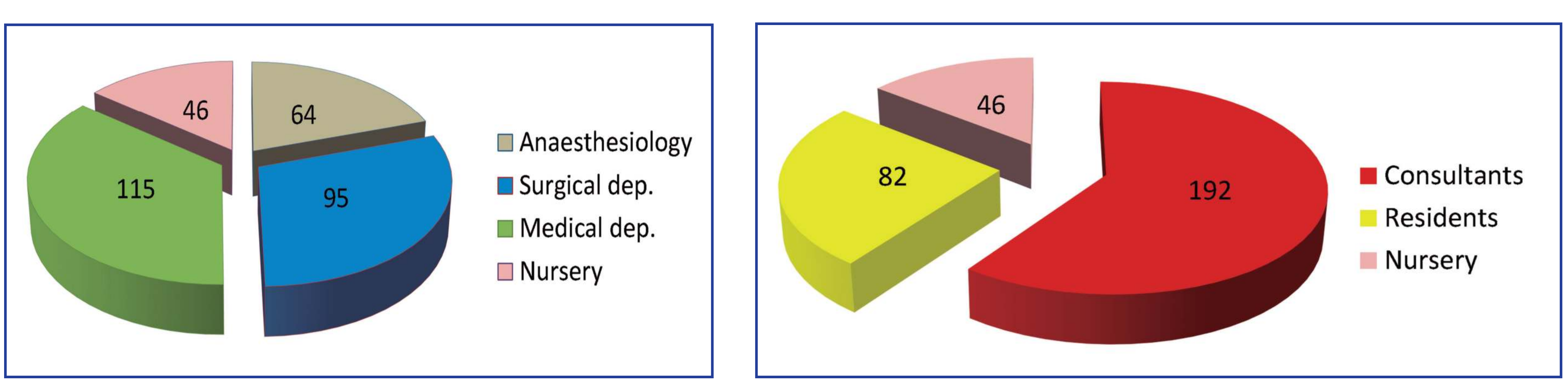

\begin{tabular}{|c|c|c|c|c|c|}
\hline & Content & $\mathrm{K}^{+}$ & $\mathrm{pH}$ & Osmolarity & $\mathrm{Mg}^{2+} / \mathrm{Ca}^{2+}$ \\
\hline NS & $\begin{array}{l}\mathrm{Na}^{+} 154 \mathrm{mmol} / \mathrm{l} \\
\mathrm{Cl} 154 \mathrm{mmol} / \mathrm{l}\end{array}$ & NO & 6 & Isotonic & NO \\
\hline $\begin{array}{c}\text { Isotonic glucose } \\
\text { saline }\end{array}$ & $\begin{array}{c}\mathrm{Na}^{+} 51 \mathrm{mmol} / \mathrm{l} \\
\mathrm{G} / \mathrm{cc} 36 \mathrm{gr} / \mathrm{l}\end{array}$ & NO & 4.5 & Isotonic & NO \\
\hline Dextrose $5 \%$ & Gluc 50gr/l & NO & 4.5 & Hypotonic & NO \\
\hline Dextrose $10 \%$ & Gluc 100gr/l & NO & 4.5 & Hypotonic & NO \\
\hline GFN $\circledast$ & $\mathrm{Na}^{+} 154 \mathrm{mmol} / \mathrm{I}$ & YES & 7 & Isotonic & YES \\
\hline PLY 7.4® & $\mathrm{Na}^{+} 140 \mathrm{mmol} / \mathrm{I}$ & YES & 7.4 & Isotonic & YES \\
\hline
\end{tabular}
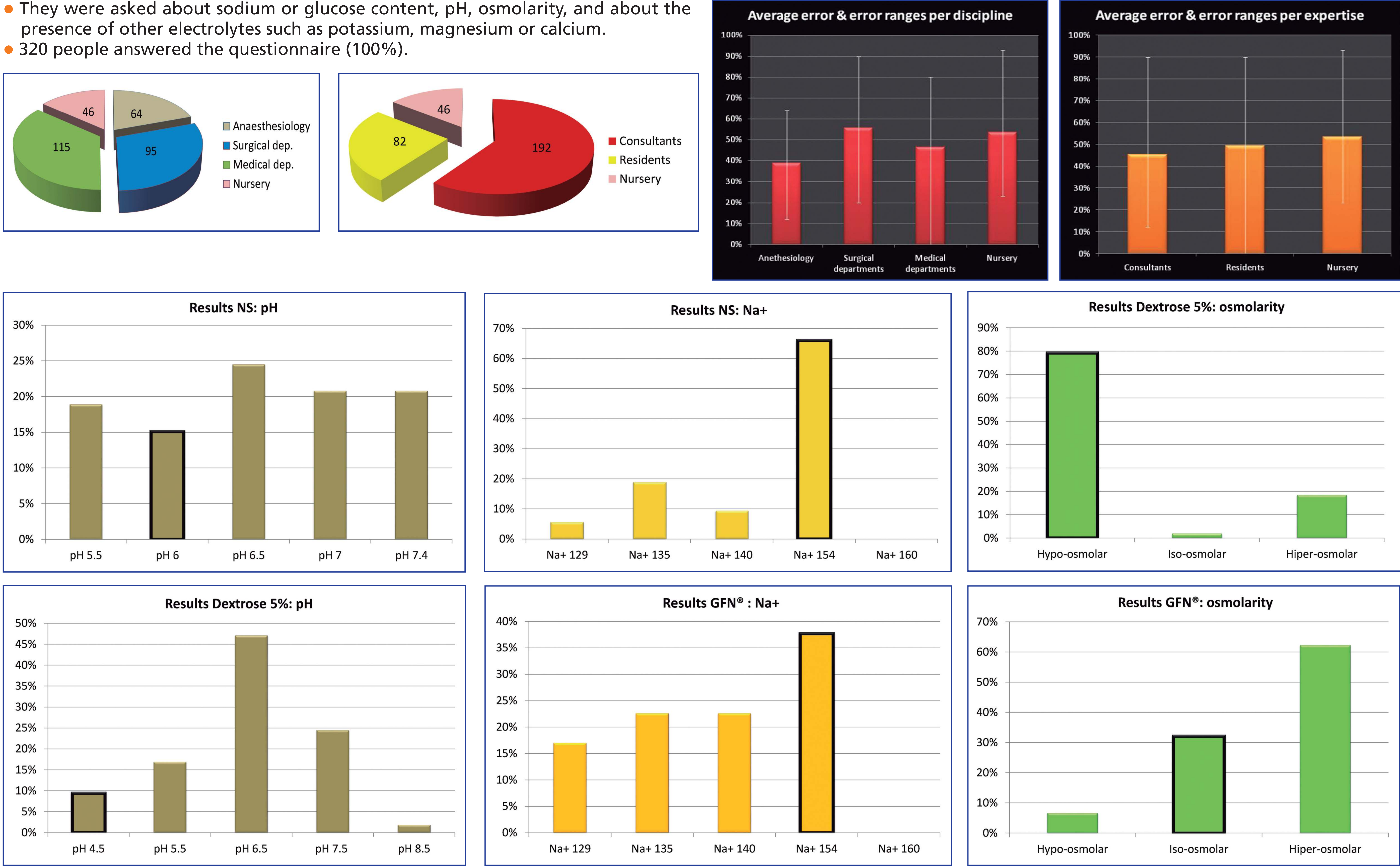

\section{Discussion}

- Healthcare providers prescribing iv solutions should know the exact composition of the iv solutions in order to avoid potentially harmful situations for the patient (pulmonary/peripheral oedema, hypo/hypernatremia, anastomotic leaks or gastrointestinal hypoperfusion, volume depletion and shock).

- There is a clear need for intra-hospital protocols on iv fluid therapy.

\section{Conclusions}

Fluid prescription should be given the same status as drug prescription.

Hospitals should establish systems to ensure that all healthcare professionals involved in prescribing iv fluid therapy are trained (fluid balance, patient needs of

fluids and risks and benefits of each fluid).

Take into account all other sources of fluid or electrolyte intake like drugs, continuous infusions, parenteral nutrition or blood products.

All patients receiving iv fluids need regular monitorying: fluid balance charts, clinical fluid status, laboratorie values and weight.

\section{References}

- Dose and type of crystalloid fluid therapy in adult hospitalized patients. Smorenberg A, et al. Perioperative Medicine. 2013,2:17. 